\title{
Gleiches mit Gleichem vergleichen ist noch lange nicht dasselbe
}

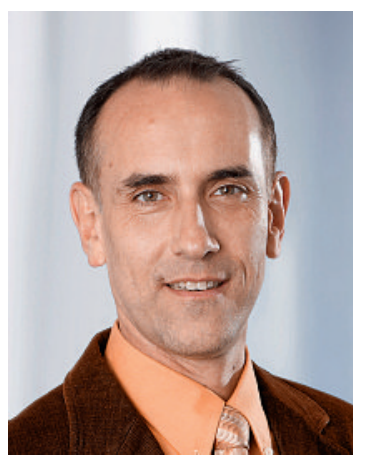

Ratings sind «in», weil sie vorgeben, eindeutige und klare Verhältnisse abzubilden. Auch die Mortalitätsraten verschiedener Spitäler scheinen prima vista für Vergleiche prädestiniert, liefern sie doch scheinbar klar die notwendigen Qualitätsindikatoren für aussagekräftige Quervergleiche. Geht man jedoch in des Pudels Kern, stellt sich heraus, dass dieser Ansatz alles andere als transparent und eindeutig ist.

Die auf Sterberaten basierenden Ratings überzeugen vielfach nicht, weil die Zahlen nicht risikoadjustiert und somit entsprechend grossen zufälligen Schwankungen unterworfen sind. Solange die jeweiligen Risikoprofile der Patienten nicht genau bekannt sind, müssen konsequenterweise Mortalitätsregister und -vergleiche mit grossem Vorbehalt interpretiert werden.

Erst unter Einbezug entsprechender Daten wie z. B. Alter, Allgemeinzustand, Co-Morbidität usw. kann man versuchen, die risikogewichtete Sterbewahrscheinlichkeit zu prognostizieren. Diese Prognose kann dann mit der effektiven Sterblichkeit verglichen werden. Anderenfalls führen die Zahlen unnötigerweise zu falscher Aufmerksamkeit und gelinde gesagt - zur Verunsicherung der Bevölkerung im allgemeinen und der Patienten im besonderen.

\section{Die Qualität von Spitälern lässt sich nicht an der Sterberate festmachen.}

Diese Problematik wurde auch in einer sehr aufschlussreichen Studie im British Medical Journal thematisiert [1]. Die beiden Epidemiologen Richard Lilford und Peter Pronovost kamen zum Schluss, dass Sterberaten sehr häufig nicht aussagekräftig für Spital-Qualitätsvergleiche sind. Die Erkenntnisse würden wenig über die eigentlichen Ursachen und Gründe für die Sterberaten aussagen, führten jedoch zu Stigmatisierung und hinterliessen bei allen Betroffenen und Beteiligten Gefühle der Hilflosigkeit und Frustration. Gründe für die Popularität von Sterberaten sehen die beiden Autoren im Bedürfnis der politischen Entscheidungsträger nach scheinbar klaren und einfach messbaren Qualitätsstandards. Die Epidemiologen plädieren für stringente und nachvollziehbare Bewertungssysteme von internen Spitalprozessen, um zukünftige Fehler zu vermeiden und um auf dieser Basis faire Vergleiche mit anderen Spitälern durchzuführen. Dies scheint mir der richtige Ansatz und kann nur unterstrichen werden.

Aussagekräftiger zur Bewertung eines Spitals sind Qualitätssysteme, die sicherstellen, dass die eigentlichen Spitalleistungen wie Diagnose, Behandlung, Pflege und Betreuung gut sind und es auch bleiben. Dazu gehören standardisierte Prozesse und somit auch die schriftlichen SOPs (standard operating procedures $=$ Arbeitsvorschriften), die z. B. durch Zertifizierungen und Akkreditierungen systematisch über-

\section{Interne Spitalprozesse sind entscheidend für eine gute Qualität.}

prüft und gewährleistet werden. Was für Spitäler und deren Vergleiche gilt, trifft auch auf die Aussagekraft von ÄrzteVergleichen zu. Die Bewertung und der Vergleich einzelner Ärzte-Leistungen an bestimmten Spitälern sind genauso wenig aussagekräftig, wenn sie zentrale Rahmenbedingungen ausklammern. In der Vielzahl der Fälle müsste die Team-Leistung bewertet und der Gesamtprozess begutachtet werden.

Ein weiteres und wichtiges Qualitätssystem stellt die medizinische Lehre dar - die Funktion als Lehrspital ist deshalb nicht zu unterschätzen. Lehre ist somit nicht «nur ein Kostenfaktor", denn wer ausbildet, ist gezwungen, sich permanent kritisch mit dem eigenen Tun auseinanderzusetzen und auf dem neuesten Stand zu bleiben.

Dass in Schweizer Spitälern prozentual mehr Menschen als sonstwo in der Schweiz sterben, ist bekannt. Das hängt aber nicht mit der medizinischen Versorgung, sondern mit der Gesundheit der Patienten zusammen: Diese Menschen sind entweder viel zu schwer krank oder zu schwer verletzt, um weiterzuleben. Es ist schön, wenn wir Ärzte den Kampf um das Leben eines Schwerkranken oder Verunfallten gewinnen, aber Sterben gehört ebenso zum Lebenskreis.

Ein Pessimist würde die grosse Gefahr von zukünftigen Spital-Ratings darin sehen, dass eventuelle Risiko-Operationen - die dann die Sterbestatistik negativ beeinflussen könnten - gar nicht erst ausgeführt und entsprechende Risiken möglichst verlegt würden. Wenn aber das Sterben in Schweizer Spitälern zu einem Qualitätsindikator wird und damit das alltägliche Sterben schlagartig zu einem Imageproblem, dann hätten wir ein wirkliches Problem!

Dr. med. Gert Printzen, Mitglied des Zentralvorstandes, Verantwortlicher Ressort Heilmittel und Ressort Angestellte Ärzte

1 Lilford R, Pronovost P. Using hospital mortality rates to judge hospital performance: a bad idea that just won't go away. BMJ. 2010;340:955-7. 